\section{Aspectos dos processos de prescrição e dispensação de medicamentos na percepção do paciente: estudo de base populacional em Fortaleza, Ceará, Brasil}

\author{
Drug prescription and dispensing from the \\ patient's perspective: a community-based study \\ in Fortaleza, Ceará State, Brazil
}

\author{
${ }^{1}$ Departamento de Farmácia, \\ Universidade Federal do \\ Ceará, Fortaleza, Brasil. \\ 2 Instituto de Saúde Coletiva, \\ Universidade Federal da \\ Bahia, Salvador, Brasil. \\ Correspondência \\ P. S. D. Arrais \\ Grupo de Prevenção ao Uso \\ Indevido de Medicamentos, \\ Departamento de Farmácia, \\ Universidade Federal do \\ Ceará. \\ Rua Nunes Valente 1427, \\ apto. 802, Fortaleza, $C E$ \\ 60125-070, Brasil. \\ parrais@ufc.br
}

\begin{abstract}
The objective of this study was to analyze aspects of the physician-patient and pharmacist-patient relationship, based on patients' opinions. It consists of community-based research performed in Fortaleza, Ceará State, Brazil, where 957 persons were interviewed; 904 answered questions about the last visit to the physician and 831 about the last visit to the pharmacy. The data reflect several aspects of medical and pharmaceutical practice and patients' attitudes towards the questions posed and information provided to guide rational use of drugs. In the therapeutic choice, physicians failed to ask their patients about hypersensitivity to drugs (65\%) and use of other medication (64.1\%) and ignored recommendations concerning adverse drug reactions (73.3\%) and drug interactions (58.2\%). The situation was even worse in hospitals, where in most cases a pharmacist's assistant was responsible for dispensing to patients (57.1\%). The study suggests poor quality in the medical and pharmaceutical roles and patient apathy towards proceedings involving the prescription and receipt of drugs in pharmacies, as well as in rational use.
\end{abstract}

Drug Prescriptions; Drug Dispensatories; Drug Utilization; Pharmacies
Paulo Sérgio Dourado Arrais 1,2

Maurício Lima Barreto 2

Helena Lutéscia Luna Coelho 1,2

\section{Introdução}

Ter acesso à assistência médica e a medicamentos não implica necessariamente em melhores condições de saúde ou qualidade de vida, pois os maus hábitos prescritivos, as falhas na dispensação, a automedicação inadequada podem levar a tratamentos ineficazes e pouco seguros. No entanto, é evidente que a possibilidade de receber o tratamento adequado, conforme e quando necessário, reduz a incidência de agravos à saúde, bem como a mortalidade para muitas doenças.

Segundo o Guia Para a Boa Prescrição Médica da Organização Mundial da Saúde (OMS) 1, após selecionar o tratamento medicamentoso e escrever a receita, o médico deve informar o paciente sobre: (a) os objetivos a curto (ou a longo) prazo do tratamento instituído; (b) como, quando e por quanto tempo deve tomar o medicamento; (c) seus benefícios e riscos (interações medicamento-medicamento ou medicamento-alimento, reações adversas, intoxicações); (d) procedimentos a seguir se surgirem alguns efeitos adversos; (e) como guardar os medicamentos; e (f) o que fazer com as sobras. Verifica-se, portanto, que o medicamento é um produto que, acompanhado de informação, facilita o seu uso correto.

Por outro lado, é responsabilidade do farmacêutico durante a dispensação: respeitar o direito do usuário de conhecer o medicamento que lhe é dispensado e de decidir sobre sua saúde e seu 
bem-estar, informar e assessorar o paciente sobre a utilização correta do medicamento ${ }^{2}$.

No Brasil e em Fortaleza, Ceará, alguns estudos evidenciam falhas na qualidade da assistência médica e farmacêutica junto aos pacientes 3,4,5,6. No caso específico da dispensação, a ausência do farmacêutico nas unidades públicas e privadas é um fator crítico para a implementação do uso racional de medicamentos 7,8.

Atualmente, verifica-se a necessidade do resgate das relações médico-paciente e farmacêutico-paciente, como um aspecto chave para a melhoria da qualidade do serviço de saúde, no qual a personalização da assistência, a humanização do atendimento e o direito à informação são componentes básicos deste processo.

Considerando o exposto acima e a falta de estudos mais amplos sobre a qualidade da assistência médica e farmacêutica no Município de Fortaleza, verificou-se a necessidade de conhecer melhor o que acontece de fato nos serviços de saúde, centrando o estudo no ato da prescrição médica e na dispensação de medicamento, no intuito de responder às seguintes questões: o médico indaga a seu paciente sobre possíveis quadros "alérgicos" ou de hipersensibilidade e sobre o uso de outros medicamentos, antes de prescrever um tratamento medicamentoso?; o médico está orientando adequadamente seus pacientes sobre a terapêutica instituída, seus benefícios e riscos?; o farmacêutico está presente nas farmácias e/ou drogarias e executa adequadamente a dispensação?; e, finalmente, qual a participação do paciente nesses processos?

O presente estudo tem como objetivo principal avaliar, a partir do ponto de vista dos usuários, aspectos do comportamento do médico, do farmacêutico e do paciente, respectivamente, durante a conduta prescritiva, no ato da dispensação e, no caso do paciente, em ambas as situações, nos setores público e privado. Os resultados subsidiarão propostas para a melhoria da qualidade da atenção à saúde.

\section{Métodos}

O presente trabalho é parte do projeto de pesquisa intitulado Epidemiologia do Consumo de Medicamentos e Reações Adversas no Município de Fortaleza (PECMEF), um estudo transversal de base populacional, que objetiva conhecer os padrões de consumo de medicamentos na população de Fortaleza e as influências de variáveis demográficas, sócio-econômicas, biológicas e de indicadores da condição de saúde e de utilização de serviços de saúde 9 .
Fortaleza é a quinta maior capital do Brasil, contendo, aproximadamente, um terço da população do Estado do Ceará. A rede de saúde municipal está organizada em seis secretarias regionais, com uma composição de 81 Centros de Saúde, oito hospitais regionais e um hospital para atendimento de urgência e emergência em traumatologia e sistema de atendimento à urgência pré-hospitalar com ambulância 24 horas 3 . A população ainda conta com outras unidades de saúde, conveniadas ou não ao Sistema Único de Saúde (SUS), e com as unidades de referência do estado. Indicadores apontam que a quantidade de recursos humanos para consultas médicas e a carga horária contratada mostram-se insuficientes, principalmente, porque muitos dos médicos estão dedicados à assistência hospitalar 10 .

A população do estudo envolve as pessoas residentes no Município de Fortaleza. O plano de amostragem foi elaborado com base em técnicas de processos probabilísticos com estratificação dos bairros e setores censitários do Instituto Brasileiro de Geografia e Estatística, conforme descrito em artigo de Arrais et al. 9. Um total de 331 domicílios foram visitados, nos quais foram encontradas 1.378 pessoas. Dessas, 1.370 foram entrevistadas, e oito foram perdas e recusas. Entretanto, apenas 957 foram selecionadas, considerando que as mesmas preenchiam os critérios de inclusão para o presente estudo: ser maior de 14 anos e ter respondido às perguntas formuladas.

A coleta de dados foi realizada entre outubro de 2002 e janeiro de 2003. As informações foram obtidas por meio de entrevista realizada no domicílio do participante. Dez acadêmicos do curso de Farmácia foram treinados para esse fim. Foi utilizado um questionário estruturado e pré-codificado para a coleta das informações. O questionário do presente estudo compõe os blocos 8 e 9 da terceira parte do questionário do PECMEF. O instrumento foi previamente testado.

As informações coletadas dizem respeito a sexo, idade, renda familiar mensal, história de doenças crônicas referidas pelo indivíduo, posse de plano privado de saúde, consulta médica nos últimos três meses, internação hospitalar nos últimos 12 meses e as práticas do médico no ato da prescrição de medicamentos, por ocasião da última visita do entrevistado ao médico, assim como as práticas de trabalho dos profissionais que dispensam medicamentos (farmacêuticos, balconistas ou outros), de acordo com a última compra (ou recebimento de medicamento) efetuada na farmácia ou drogaria.

Com relação à prática médica, foram coletadas as seguintes informações: data da última visita, local da consulta (setor público ou privado) 
e se houve prescrição de algum medicamento. Caso afirmativo, perguntava-se sobre a conduta antes e depois da emissão da receita. Foi questionado se, antes de realizar a prescrição, o médico fazia perguntas ao paciente sobre possível alergia a algum tipo de medicamento e se estava fazendo uso de outros medicamentos. Foi avaliado também se, após a emissão da prescrição, o médico havia orientado quanto à forma correta de tomar o medicamento, os possíveis problemas e efeitos adversos que o medicamento poderia causar, sua interação com outros medicamentos e a importância de cumprir o tratamento até o fim. Ainda com relação ao ato pós-prescrição e no caso de as pessoas terem respondido negativamente aos questionamentos, era perguntado aos mesmos se eles tomavam a iniciativa de perguntar ao médico sobre os assuntos explorados.

Com relação à prática da dispensação de medicamentos nas farmácias ou drogarias, foram coletadas informações sobre: data da última visita, local da compra ou aquisição (setor público ou privado), responsável pelo atendimento (farmacêutico, balconista, outros), e se o(s) medicamento(s) procurado(s) havia(m) sido indicado(s) por médico. Também foi perguntado se o dispensador havia feito perguntas sobre se o paciente era alérgico a algum tipo de medicamento e se estava fazendo uso de outros medicamentos, assim como foi explorado se o mesmo havia orientado quanto à forma correta de tomar o medicamento, os possíveis problemas que o medicamento poderia causar, sua interação com outros medicamentos, a importância de cumprir o tratamento até o fim e os cuidados especiais no armazenamento (guarda) do produto em casa. No caso de respostas negativas, era perguntado às pessoas entrevistadas se elas tomavam a iniciativa de perguntar ao dispensador sobre os assuntos explorados. É importante lembrar que existe a possibilidade de a pessoa ter ido comprar ou receber medicamento para si ou para outros.

Os dados foram armazenados e analisados por meio do programa Epi Info, versão 6.0 (Centers for Disease Control and Prevention, Atlanta, Estados Unidos). O plano de análise dos dados incluiu: uma análise descritiva através da distribuição de freqüências das variáveis do estudo e estratificação das mesmas pelo tipo de sistema de saúde utilizado, público ou privado, e, no caso da dispensação, por quem atendeu na farmácia (farmacêutico e "outros"). O teste do qui-quadrado foi utilizado para verificar as diferenças de proporções, com nível de significância $\mathrm{p}<0,05$.

A pesquisa foi aprovada pelo Comitê de Ética em Pesquisa do Complexo Hospitalar da Universidade Federal do Ceará.

\section{Resultados}

Foram entrevistados 957 indivíduos com mais de 14 anos de idade. As mulheres constituíam maioria (56,5\%). Quanto à idade dos entrevistados (faixa de variação entre 15 e 84 anos), 60,6\% tinham de 20 a 49 anos; $16,8 \%$, de 50 a 64 anos; $14,2 \%$ de 15 a 19 anos; e $8,4 \%$ de 65 ou mais anos. Uma parte considerável dos indivíduos (51,2\%) tinha renda familiar mensal de três ou mais salários mínimos; $48,1 \%$, até três salários mínimos; e $0,7 \%$ não tinha renda ou não a declarou $(n=3)$. 20,5\% eram doentes crônicos; $29,4 \%$ possuíam plano de saúde; $43,7 \%$ haviam realizado uma ou mais consultas nos últimos três meses; $11,5 \%$ haviam estado internados uma ou mais vezes nos últimos 12 meses.

Com relação à data da última consulta, $23,4 \%$ dos indivíduos visitaram o médico há menos de trinta dias da realização da entrevista; $14,1 \%$, entre um e dois meses; $12 \%$, entre dois e quatro meses; $8,7 \%$, entre quatro e seis meses; e $36,4 \%$, entre seis ou mais meses. Dez pessoas não lembraram a data da última visita médica, e 41 não responderam ou referiram nunca ter ido ao médico. Ao final, 916 pessoas responderam às questões sobre a última consulta médica.

Na Tabela 1, pode-se observar a distribuição dos dados relativos à experiência com a última consulta médica, conforme o local da consulta, prescrição de medicamentos, a ocorrência de indagação médica sobre alergia anterior e uso de outros medicamentos e a freqüência da orientação médica sobre como tomar o medicamento, os cuidados com as reações adversas e interações medicamentosas e a importância de cumprir o tratamento.

A maioria dos entrevistados visitaram o médico no setor público (60,5\%). Em 56,4\% das visitas, o paciente saiu do consultório com uma prescrição médica. Os pacientes do setor público receberam mais prescrição de medicamentos do que os do setor privado (Tabela 1). Essa diferença foi estatisticamente significante $(\mathrm{p}<$ 0,05).

Quanto ao ato médico que precedeu a indicação de medicamentos, 35\% e 35,9\% dos indivíduos informaram, respectivamente, que o médico fez pergunta sobre manifestações anteriores de quadros de alergia por medicamento e sobre a possibilidade de o indivíduo estar, ou não, usando outras medicações (Tabela 1). Os pacientes do setor privado foram mais questionados que os do setor público, diferença estatisticamente significante $(\mathrm{p}<0,05)$.

Quanto ao processo de orientação e informação sobre os medicamentos (Tabela 1), observou-se que os médicos orientaram em $92,5 \%$ das 
Experiência com a última consulta médica (N = 904) *. Fortaleza, Ceará, Brasil, 2002/2003.

\begin{tabular}{|c|c|c|c|c|c|c|c|}
\hline \multirow[t]{2}{*}{ Variáveis } & \multicolumn{2}{|c|}{ Total } & \multicolumn{2}{|c|}{ Público } & \multicolumn{2}{|c|}{ Privado } & \multirow[t]{2}{*}{$\mathrm{p}$ valor } \\
\hline & $\mathbf{n}$ & $\%$ & $\mathbf{n}$ & $\%$ & $\mathbf{n}$ & $\%$ & \\
\hline \multicolumn{8}{|c|}{ Prescrição no final da consulta $(\mathrm{N}=904)$ * } \\
\hline Sim & 510 & 56,4 & 335 & 60,5 & 175 & 50,0 & $<0,05$ \\
\hline \multicolumn{8}{|c|}{ Durante a consulta, o médico perguntou sobre $(\mathrm{N}=510)$ ** } \\
\hline \multicolumn{8}{|c|}{ a) Alergia anterior } \\
\hline Sim & 178 & 35,0 & 95 & 28,4 & 83 & 47,4 & $<0,05$ \\
\hline \multicolumn{8}{|c|}{ b) Uso de outros medicamentos } \\
\hline Sim & 183 & 35,9 & 105 & 31,3 & 78 & 44,6 & $<0,05$ \\
\hline \multicolumn{8}{|c|}{ O médico orientou sobre $(N=510)$ ** } \\
\hline \multicolumn{8}{|c|}{ a) Como tomar o medicamento } \\
\hline Sim & 472 & 92,5 & 306 & 91,3 & 166 & 94,9 & 0,151 \\
\hline \multicolumn{8}{|c|}{ b) Reações adversas } \\
\hline $\operatorname{Sim}$ & 136 & 26,7 & 75 & 22,4 & 61 & 34,9 & $<0,05$ \\
\hline \multicolumn{8}{|c|}{ c) Interações medicamentosas } \\
\hline $\operatorname{Sim}$ & 213 & 41,8 & 143 & 42,7 & 70 & 40,0 & 0,056 \\
\hline \multicolumn{8}{|c|}{ d) Importância de cumprir o tratamento } \\
\hline Sim & 360 & 70,6 & 227 & 67,8 & 133 & 76,0 & 0,053 \\
\hline
\end{tabular}

* Exclui os casos que não lembram ( $n=12)$;

** Incluem as pessoas que, no item anterior, disseram ter recebido prescrição no final da consulta.

ocasiões sobre como tomar o medicamento, em $70,6 \%$ sobre a importância de cumprir o tratamento, em $26,7 \%$ das situações informaram sobre as possibilidades de surgimento de reações adversas durante o tratamento e em $41,8 \%$ sobre a possibilidade de ocorrência de interações medicamentosas. Os pacientes do setor privado foram mais informados sobre como tomar o medicamento, reações adversas e importância de cumprir o tratamento do que os do setor público. No caso das reações adversas, a diferença foi estatisticamente significante $(p<0,05)$ e, no caso de como tomar o medicamento, ficou no limite da significância.

Nos casos nos quais não ocorreu o repasse das informações/orientações por parte dos médicos, os pacientes, também, não tomaram a iniciativa de perguntar, na maioria das vezes (Tabela 2 ).

Com relação à data da última compra ou recebimento na farmácia, $41,5 \%$ dos indivíduos visitaram a farmácia/drogaria há menos de trinta dias da realização da entrevista; $15 \%$, entre um e dois meses; $11,5 \%$, entre dois e quatro meses; $5,2 \%$, entre quatro e seis meses; e $13 \%$, entre seis ou mais meses. Oito pessoas não lembraram a data da última visita, 63 não responderam às perguntas, 23 informaram que a compra ou recebimento era atribuição de outra pessoa, e 38 indivíduos referiram nunca terem ido à farmácia.
Ao final, 833 pessoas responderam às questões sobre a última aquisição de medicamentos na farmácia ou drogaria.

Na Tabela 3, pode-se observar a distribuição dos dados relativos à experiência com a última dispensação de medicamentos na farmácia ou drogaria, de acordo com o local da dispensação, dispensação com apresentação de prescrição e perguntas sobre alergia anterior e uso de outros medicamentos.

A dispensação ocorreu, principalmente, no setor privado (86,6\%), tendo o balconista como o maior responsável pela mesma $(57,1 \%)$, seguido do farmacêutico (23,6\%) e outros $(19,2 \%)$ (Tabela 3). Entre os "outros", destaca-se o uso da via telefônica para a aquisição do medicamento $(74,4 \%)$, prática restrita ao setor privado, seguido do enfermeiro $(n=2)$, médico $(n=2)$ e estagiário $(n=$ 4). Trinta e dois indivíduos não lembram quem realizou a dispensação (20\%).

Em $67 \%$ das situações, houve dispensação de produtos prescritos por médicos. Durante o processo da dispensação, o dispensador só perguntou sobre a possibilidade de o indivíduo ter tido algum quadro alérgico na vida ou se ele estava tomando outros medicamentos, respectivamente, em $9,6 \%$ e $9,9 \%$ das situações (Tabela 3 ). As pessoas que atenderam no setor público fizeram mais perguntas sobre o uso de outros medica- 
Experiência com a última dispensação de medicamentos na farmácia ou drogaria ( $N=833$ ). Fortaleza, Ceará, Brasil, $2002 / 2003$.

\begin{tabular}{|c|c|c|c|c|c|c|c|}
\hline \multirow[t]{2}{*}{ Variáveis } & \multicolumn{2}{|c|}{ Total } & \multicolumn{2}{|c|}{ Público } & \multicolumn{2}{|c|}{ Privado } & \multirow[t]{2}{*}{$\mathrm{p}$ valor } \\
\hline & $\mathrm{n}$ & $\%$ & $\mathrm{n}$ & $\%$ & $\mathrm{n}$ & $\%$ & \\
\hline \multicolumn{8}{|l|}{ Dispensador ( $N=833)$} \\
\hline Farmacêutico & 198 & 23,8 & 37 & 33,0 & 161 & 22,3 & $<0,05$ \\
\hline \multicolumn{8}{|c|}{ Durante a dispensação, o dispensador } \\
\hline \multicolumn{8}{|c|}{ perguntou sobre $(\mathrm{N}=831)$ * } \\
\hline \multicolumn{8}{|c|}{ a) Alergia anterior } \\
\hline Sim & 80 & 9,6 & 16 & 14,4 & 64 & 8,9 & 0,066 \\
\hline \multicolumn{8}{|c|}{ b) Uso de outros medicamentos } \\
\hline Sim & 82 & 9,9 & 18 & 16,2 & 64 & 8,9 & 0,016 \\
\hline \multicolumn{8}{|c|}{ O dispensador orientou sobre $(\mathrm{N}=831)$ * } \\
\hline \multicolumn{8}{|c|}{ a) Como tomar o medicamento } \\
\hline $\operatorname{Sim}$ & 214 & 25,8 & 45 & 40,5 & 169 & 23,5 & $<0,05$ \\
\hline \multicolumn{8}{|l|}{ b) Reações adversas } \\
\hline Sim & 65 & 7,8 & 16 & 14,4 & 49 & 6,8 & $<0,05$ \\
\hline \multicolumn{8}{|c|}{ c) Interações medicamentosas } \\
\hline $\operatorname{Sim}$ & 119 & 14,3 & 25 & 22,5 & 94 & 13,1 & $<0,05$ \\
\hline \multicolumn{8}{|c|}{ d) Importância de cumprir o tratamento } \\
\hline Sim & 137 & 16,5 & 32 & 28,8 & 105 & 14,6 & $<0,05$ \\
\hline \multicolumn{8}{|c|}{ e) Cuidados com armazenamento } \\
\hline Sim & 114 & 13,7 & 28 & 25,2 & 86 & 11,9 & $<0,05$ \\
\hline
\end{tabular}

* Em todas as variáveis, existem dois casos de pessoas que foram retiradas da presente análise por não lembrarem.

mentos do que as do setor privado, e essa diferença foi significante $(\mathrm{p}<0,05)$.

Quanto à orientação sobre como tomar o medicamento (Tabela 3), a possibilidade de surgir reações adversas, os cuidados com a interação medicamentosa, a importância de cumprir o tratamento e cuidados com o armazenamento, não houve, na maioria das vezes, a orientação dos pacientes ou de seus responsáveis, respectivamente, em $74,2 \%, 92,2 \%, 85,7 \%, 83,5 \%, 86,3 \%$ dos casos.

Observou-se também que, na maioria, os pacientes e/ou seus representantes não manifestaram nenhum interesse em perguntar sobre os assuntos (Tabela 4). Em todas as situações, o serviço público prestou mais orientações do que o setor privado, sendo as diferenças estatisticamente significantes $(p<0,05)$, em todos os itens avaliados.

Na estratificação por quem atendeu na farmácia, observou-se que o farmacêutico pergunta e orienta mais do que os "outros" atendentes. A diferença foi significante para todas as variáveis exploradas no estudo $(\mathrm{p}<0,05)$. Entretanto, quando se estratifica o atendimento por setor, público e privado, observa-se que, no setor público, o farmacêutico faz mais perguntas e orienta mais comparado aos "outros", mas as diferenças não são significantes. Já no caso do setor privado, a situação é a mesma, exceto no caso da orientação sobre reações adversas, mas as diferenças são estatisticamente significantes $(\mathrm{p}<0,05)$.

\section{Discussão}

Considerando que apenas $29,4 \%$ dos entrevistados possuíam plano de saúde, era de se esperar que boa parte das pessoas buscassem assistência médica no setor público (60,5\%). No Brasil, estima-se que $25 \%$ da população possua plano de saúde e que mais de $90 \%$ utilizam o SUS 11 . É provável que as transformações sociais e econômicas pelas quais passa o país, em que existe uma nítida perda de poder aquisitivo das famílias aliada aos altos preços dos planos de saúde, tenham influenciado na busca de assistência no setor público. Em nível local, a elevada procura pelo SUS também foi constatada por Correia $4 \mathrm{e}$ Fernandes 12, que, em seus estudos, registraram, respectivamente, proporções de $96 \%$ e 69,1\%.

Uma parte considerável das pessoas que foram à consulta médica saíram com uma prescrição (56,4\%). A elevada prescrição medicamen- 
Distribuição do número de indivíduos que não receberam orientação do médico ou do dispensador sobre como tomar o medicamento, cuidados com reações adversas e interações medicamentosas, importância de cumprir o tratamento e cuidados com armazenagem dos medicamentos e as respectivas freqüências do interesse do paciente em perguntar sobre os assuntos ao médico e ao dispensador que o atende. Fortaleza, Ceará, Brasil, 2002/2003.

\begin{tabular}{|c|c|c|}
\hline & n & $\%$ \\
\hline \multicolumn{3}{|c|}{ O paciente tomou a iniciativa de perguntar ao médico sobre } \\
\hline \multicolumn{3}{|c|}{ a) Como tomar o medicamento $(\mathrm{N}=38)$ * } \\
\hline Sim & 6 & 15,8 \\
\hline Não & 32 & 84,2 \\
\hline \multicolumn{3}{|c|}{ b) Reações adversas $(N=374)$ * } \\
\hline Sim & 27 & 7,2 \\
\hline Não & 347 & 92,8 \\
\hline \multicolumn{3}{|c|}{ c) Interações medicamentosas ( $N=297)$ * } \\
\hline Sim & 6 & 2,0 \\
\hline Não & 291 & 98,0 \\
\hline \multicolumn{3}{|c|}{ d) Importância de cumprir o tratamento $(N=150)$ * } \\
\hline Sim & 1 & 0,7 \\
\hline Não & 149 & 99,3 \\
\hline \multicolumn{3}{|c|}{ O paciente tomou a iniciativa de perguntar ao dispensador sobre: } \\
\hline \multicolumn{3}{|c|}{ a) Como tomar o medicamento $(\mathrm{N}=617)$ * } \\
\hline Sim & 18 & 2,9 \\
\hline Não & 599 & 97,1 \\
\hline \multicolumn{3}{|c|}{ b) Reações adversas $(N=766)$ * } \\
\hline Sim & 12 & 1,6 \\
\hline Não & 754 & 98,4 \\
\hline \multicolumn{3}{|c|}{ c) Interações medicamentosas ( $\mathrm{N}=712)$ * } \\
\hline Sim & 7 & 1,0 \\
\hline Não & 705 & 99,0 \\
\hline \multicolumn{3}{|c|}{ d) Importância de cumprir o tratamento $(\mathrm{N}=694)$ * } \\
\hline Sim & 3 & 0,4 \\
\hline Não & 691 & 99,6 \\
\hline \multicolumn{3}{|c|}{ e) Cuidados com armazenamento $(\mathrm{N}=717)$ * } \\
\hline Sim & 1 & 0,1 \\
\hline Não & 716 & 99,9 \\
\hline
\end{tabular}

* Número de pacientes que não receberam orientação do médico ou do dispensador sobre o assunto em questão.

tosa após uma consulta médica parece ser fato rotineiro em Fortaleza e em outra microrregião do Ceará (Quixadá). É o que constatam Correia 4 e Fernandes 12, quando identificam freqüências elevadas de prescrição após consultas médicas, respectivamente, $90 \%$ e $87,9 \%$. Segundo Correia 4 , esse elevado percentual pode denotar baixa qualidade no atendimento ou interesse do profissional em finalizar a consulta, saltando etapas precedentes essenciais de exame do paciente e formulação de um diagnóstico.

O estudo evidenciou que, durante a consulta, o médico não perguntava aos pacientes sobre alergias medicamentosas anteriores (65\%) e uso de outros medicamentos (64,1\%). Quando o paciente vai ao médico, existe a possibilidade concreta de já ter apresentado hipersensibilidade a algum fármaco e estar tomando medicamentos adquiridos sem receita, oferecidos por amigos e parentes, balconistas de farmácia, ou até mesmo por outros médicos. Portanto, obter essas informações é de fundamental importância para melhor direcionar a escolha de nova terapêutica, evitando expor o paciente a riscos desnecessários. A situação deve ser observada, principalmente, entre a população idosa, pois são os mais susceptíveis à polimedicação e, conseqüentemente, aos efeitos das interações medicamentosas e redundâncias terapêuticas 13 . 
Distribuição dos dados relativos à experiência com a última dispensação de medicamentos na farmácia ou drogaria, segundo orientação do dispensador sobre como tomar o medicamento, cuidados com as reações adversas e interações medicamentosas, importância de cumprir o tratamento, cuidados com o armazenamento do medicamento e, no caso negativo, iniciativa do paciente em perguntar. Fortaleza, Ceará, Brasil, 2002/2003.

\begin{tabular}{|c|c|c|}
\hline & $\mathbf{n}$ & $\%$ \\
\hline \multicolumn{3}{|c|}{ O paciente tomou a iniciativa de perguntar ao dispensador sobre * } \\
\hline \multicolumn{3}{|c|}{ a) Como tomar o medicamento $(\mathrm{N}=617)$} \\
\hline Sim & 18 & 2,9 \\
\hline Não & 599 & 97,1 \\
\hline \multicolumn{3}{|c|}{ b) Reações adversas $(N=766)$} \\
\hline Sim & 12 & 1,6 \\
\hline Não & 754 & 98,4 \\
\hline \multicolumn{3}{|c|}{ c) Interações medicamentosas ( $N=712$ ) } \\
\hline $\operatorname{Sim}$ & 7 & 1,0 \\
\hline Não & 705 & 99,0 \\
\hline \multicolumn{3}{|c|}{ d) Importância de cumprir o tratamento $(\mathrm{N}=694)$} \\
\hline Sim & 3 & 0,4 \\
\hline Não & 691 & 99,6 \\
\hline \multicolumn{3}{|c|}{ e) Cuidados com armazenamento ( $\mathrm{N}=717$ ) } \\
\hline Sim & 1 & 0,1 \\
\hline Não & 716 & 99,9 \\
\hline
\end{tabular}

* Em todas as variáveis, existem dois casos em que as pessoas não lembram, o que corresponde a 0,2\% do total de cada variável.

O fato de os médicos do setor privado perguntarem mais sobre alergias e uso de outros medicamentos em comparação com os do setor público pode estar relacionado com a maior demanda de serviços enfrentada pelo setor público, o que inviabiliza a disponibilidade de um atendimento mais racional e humanizado. No setor privado, a oferta de serviço é maior, e os médicos lidam com um público diferenciado, com maior nível educacional, poder aquisitivo, e, portanto, têm maior satisfação e interesse de prestar melhor atendimento para satisfazer e garantir a sua clientela. Essa disparidade no atendimento entre os dois setores também foi observado por Correia 4 . O autor verificou que, entre as pessoas que possuíam plano de saúde, $54 \%$ haviam recebido diagnóstico, exame físico, tratamento e alguma orientação, e apenas um terço dos usuários do SUS recebeu igual orientação.

No que diz respeito à avaliação da comunicação médico-paciente durante a conduta prescritiva, observou-se que o médico orientou a maioria dos pacientes a tomar o medicamento prescrito $(92,5 \%)$ e a cumprir adequadamente o tratamento $(70,6 \%)$, menosprezando, na maioria das vezes, os cuidados com as possíveis reações adversas e interações medicamentosas. Apesar das orientações sobre como tomar o medica- mento e a importância de cumprir o tratamento até o final, imprescindíveis ao cumprimento do esquema terapêutico, verifica-se que a assistência médica prestada à população pesquisada deixa a desejar, pois não leva em consideração algumas recomendações da OMS para a boa prescrição médica 1, fere o artigo 59 do Código de Ética Médica 14 e está permeada pelo relacionamento paternalista, no qual o médico seleciona o tratamento e as informações suficientes para o paciente aderir às suas recomendações.

Esse parece ser um problema comum nos serviços de saúde. Em estudo realizado por Franco 15 sobre a relação médico-paciente no Programa Saúde da Família (PSF) de três municípios da Bahia, verificou-se que a participação do paciente na consulta e na formulação do plano de tratamento não foi estimulada pelos médicos, e que a explicação sobre o seu problema de saúde não era aprofundada, deixando-se de informar, inclusive, quanto ao prognóstico e curso do problema. Os médicos também não verificavam a compreensão do paciente acerca do que fora exposto quanto à sua condição de saúde e sua concordância quanto ao tratamento.

A escassez de tempo e a falta de uma sensibilização prévia, desenvolvida durante a formação do médico, são apontadas por Baos 16 como res- 
ponsáveis pelo fato de o paciente ser pouco informado e consultado sobre os aspectos que interessam à sua própria saúde. Esse tipo de comportamento dentro da política atual de saúde pública é extremamente prejudicial para as intervenções em áreas estratégicas, como, por exemplo, hipertensão, diabetes, tuberculose, hanseníase e AIDS, nas quais a falta de informação e orientação pode resultar no abandono do tratamento. A própria prevenção das doenças tem sido negligenciada, em alguns casos, nas consultas médicas. É o que observa Piccini \& Victora 6 , em estudo realizado, em Pelotas, Rio Grande do Sul, sobre o manejo da hipertensão arterial sistêmica. No artigo, os autores relatam que, em muitas situações (> 50\%), os médicos não haviam recomendado aos pacientes a realização de exercícios, o abandono do tabagismo e a redução do peso corporal, considerados importantes no combate às doenças cardiovasculares.

Segundo Silva 17 (p. 18), o profissional de saúde é visto como "o promotor da reflexão necessária que poderá motivar o paciente a buscar novamente a sua saúde". Para alcançar esse objetivo, é necessário que ele esteja bem consigo próprio, no que diz respeito à sua qualidade de vida $\mathrm{e}$ às relações interpessoais gerais, o que o deixará mais sensível ao sofrimento do outro. Entretanto, nas duas últimas décadas, os trabalhadores da área de saúde no Brasil experimentaram mudanças importantes nas condições de trabalho, como os baixos salários, o pluriemprego, a flexibilização dos contratos de trabalho e a perda das garantias laborais 18. Essas mudanças, portanto, associadas à falta de infra-estrutura adequada dos serviços de saúde, principalmente públicos, têm refletido diretamente na relação médicopaciente.

O estudo também constata que a maioria dos pacientes não se interessa em fazer perguntas sobre os assuntos pesquisados. Ao não tomar essa iniciativa, o paciente corre o risco de sofrer as conseqüências do mau atendimento ou da má utilização do medicamento. O "silêncio" pode ter várias explicações, entre elas, o médico não oferecer espaço para perguntas, o paciente ficar inibido ou ter medo de fazê-las ou perceber a pressa do médico, anulando-se ${ }^{19}$. O baixo nível cultural é outro fator a ser levado em consideração. No contexto geral, os médicos não incentivam falas (não perguntam), não introduzem temas (não informam) ou não facilitam temas.

$\mathrm{Na}$ atualidade, é notória a necessidade de construção da autonomia dos pacientes, a fim de que eles reconheçam suas condições para controlar e transformar as circunstâncias da vida, pois o interesse e a segurança em si mesmo são indispensáveis para a sua cura. A procura por informações nos Centros de Informações sobre Medicamentos confirma que os pacientes estão se conscientizando, cada vez mais, acerca de seus direitos de serem informados e de participarem das escolhas que dizem respeito à sua saúde 5 .

Com relação à pouca atenção dada pelos médicos na transmissão de informações sobre reações adversas, Baos 16 alerta que os efeito adversos leves e transitórios podem influenciar na adesão ao tratamento e ser causa de abandono, logo no início, de medicamentos necessários e adequados. É importante, portanto, informar e discutir com o paciente sobre reações adversas, o que melhorará a qualidade no uso dos medicamentos, sobretudo quando se tratar de tratamentos prolongados, nos quais a taxa de abandono pode ser alta. Bakirtzief 20, em seu estudo sobre a não-adesão ao tratamento poliquimioterápico da hanseníase, compartilha da mesma opinião, salientando, ainda, que a adesão ao tratamento está associada à satisfação com o atendimento do profissional.

Também, faz-se necessário conscientizar os médicos que os pacientes necessitam de orientação clara sobre os riscos da administração concomitante de vários fármacos. Cuidados especiais devem ser dispensados, principalmente para os pacientes que utilizam anticoagulantes, hipoglicemiantes orais, digitálicos, psicotrópicos, inibidores da monoaminoxidase e fármacos citotóxicos.

No que diz respeito às informações sobre a última compra ou aquisição de medicamentos em farmácias ou drogarias, verificou-se que a maioria dos entrevistados adquiriram seus medicamentos no setor privado (86,6\%), ou seja, tiveram de comprá-los, apesar do indicativo de que parte significativa das pessoas procuraram assistência médica no serviço público.

O acesso a medicamentos é um dos principais problemas do SUS 4,11,12. O Instituto Brasileiro de Defesa do Consumidor 7 avaliou o abastecimento de medicamentos essenciais distribuídos gratuitamente pelo SUS, em 50 unidades de saúde de 11 cidades brasileiras, e constatou um quadro de grave desabastecimento de medicamentos essenciais. Em média, apenas 55,4\% dos remédios pesquisados estavam disponíveis. Em Fortaleza, estudos realizados pela Célula de Assistência Farmacêutica da Prefeitura Municipal de Fortaleza mostram que o abastecimento é irregular 21. Não existem dados para 2002, mas, a partir dos dados de janeiro de 2003, nos quais se observa uma queda brusca no nível de cobertura (50,3\%), pode-se presumir que existiram sérios problemas de abastecimento no período de realização da pesquisa, o que, de certa forma, justificaria, a grande aquisição no setor privado. 
Na cadeia do medicamento, o farmacêutico é o último elo de contato do sistema de saúde com o cliente, que anteriormente havia buscado o médico para tratar de seu problema de saúde, a quem compete a distribuição/aquisição do medicamento prescrito para tal. É, portanto, "uma das últimas oportunidades de identificar, corrigir ou reduzir possíveis riscos associados à terapêutica" 22 (p. 819).

No estudo, observou-se que a dispensação é efetuada, principalmente, pelo balconista $(57,1 \%)$. A ausência do farmacêutico no processo da dispensação é preocupante, pois a dispensação "é o ato profissional farmacêutico de proporcionar um ou mais medicamentos a um paciente, geralmente como resposta à apresentação de uma receita elaborada por um profissional autorizado", no qual ele "informa e orienta o paciente sobre o uso adequado do medicamento" 23 (p. 34). Os resultados também podem refletir a ausência do profissional no estabelecimento. É o que constata o estudo realizado pela Agência Nacional de Vigilância Sanitária (ANVISA) 8, nas farmácias/ drogarias de Fortaleza (setor privado), nos meses de julho, novembro e dezembro de 2002 e março de 2003. A ausência do profissional farmacêutico oscilou entre 68,9 e $75 \%$ e era maior entre as redes de farmácias/drogarias, variando entre 82 e $89 \%$. No setor público, só $20 \%$ das unidades de dispensação municipais possuem farmacêuti$\cos ^{3}$. A Lei n ${ }^{\circ} .5 .99124$ é clara, enquanto a farmácia ou drogaria estiver aberta deverá ter um ou mais farmacêuticos a disposição dos seus clientes.

De maneira geral, o estudo sugere que o atendimento na farmácia ou drogaria deixa muito a desejar, pois não existe, na maioria das vezes, preocupação dos dispensadores em solicitar informações importantes que possam ser utilizadas na prevenção de possíveis agravos à saúde do paciente, ocasionados pelas reações adversas e interações medicamentosas, assim como não houve a preocupação de orientar e informar sobre o tratamento, precauções e guarda do medicamento. Entretanto, verificou-se que os farmacêuticos são profissionais importantes nesse processo da dispensação, pois orientam e perguntam mais do que os "outros" atendentes, seja no setor público ou privado.

O estudo também sugere que a participação do paciente no processo da dispensação é nula. Outro aspecto interessante é o uso da via telefônica para solicitação de medicamentos e sua entrega em domicílio, fato comum em nosso país. Utilizando-se desse mecanismo, poucos foram os pacientes perguntados (entre 5 e 8) e orientados (entre 2 e 17) dentro dos aspectos abordados na pesquisa, demonstrando que o paciente ganha na comodidade, mas corre riscos por falta de orientação. O paciente, na maioria das vezes (acima de 97\%), não toma iniciativa de fazer nenhum tipo de pergunta.

Os governantes e os cidadãos têm vários motivos para acreditarem na importância do trabalho desempenhado pelos farmacêuticos, já que eles podem, entre outras ações, coibir as práticas não convencionais ou antiéticas do mercado, como a empurroterapia, as vendas comissionadas, a receptação e comercialização de cargas roubadas, a venda de medicamentos falsificados, controlar a venda indiscriminada de produtos de controle especial (Portaria no. 34425 de 12 de maio de 1998) e de antibióticos, a falta de condições sanitárias do estabelecimento, a automedicação, evitar a ocorrência de trocas e erros na entrega de medicamentos, devido à ilegibilidade das receitas médicas e/ou outras. A presença do farmacêutico é muito importante, inclusive para orientar a substituição dos medicamentos de marca pelos genéricos.

O presente trabalho não tinha a intenção de avaliar o conhecimento do paciente a respeito do medicamento prescrito, mas saber até que ponto os médicos, e de forma complementar, os farmacêuticos, estão interagindo corretamente com os pacientes, para garantir o uso correto dos medicamentos. Por outro lado, limitou-se a coletar informações da última consulta médica. Não se sabe, portanto, quantas vezes o paciente freqüentou o consultório do mesmo médico e se foi atendido por profissionais do PSF.

É provável que as distâncias entre as datas das últimas visitas ao médico e à farmácia/drogaria e a realização da entrevista possam ter influenciado as respostas das pessoas, já que $57,1 \%$ dos entrevistados consultaram o médico há dois meses ou mais; e 29,7\%, as farmácias/drogarias. É possível, também, que a última aquisição de medicamentos em farmácia ou drogaria não tenha nenhuma relação com a prescrição ocorrida durante a última consulta médica e que o farmacêutico referido nas entrevistas possa ser, na verdade, o balconista, o que limitaria algumas das considerações relatadas.

Os tipos de perguntas utilizadas e a metodologia empregada limitaram a possibilidade de fazer comparações com outros trabalhos.

\section{Conclusão}

O estudo sugere que existe uma baixa qualidade no atendimento médico e farmacêutico no $\mathrm{Mu}$ nicípio de Fortaleza.

Os achados do estudo revelam total dissonância do processo de realização de uma correta prescrição e orientação do paciente, assim como 
dispensação na farmácia e/ou drogaria, com o preconizado pela OMS ${ }^{1}$ e pelo Código de Ética Farmacêutica ${ }^{2}$ e Código de Ética Médica 14.

É necessário, portanto, que ocorram mudanças na formação médica e farmacêutica, a fim de que os aspectos éticos, a humanização do atendimento, o compromisso social e a comunicação efetiva sejam levados em consideração. A inserção, desde cedo, dos futuros profissionais na realidade do SUS, por meio de vivência e estágio, é um dos caminhos e está sendo incentivada pelo governo 26 . Os problemas com a estrutura de funcionamento dos serviços de saúde públicos, a remuneração dos profissionais e a disponibilidade de recursos humanos também são temas que não podem ser ignorados pelos gestores. Os profissionais também deverão trabalhar em equipe e considerar a sabedoria popular nas atividades de educação em saúde.

Aos profissionais no exercício da medicina, é notória a necessidade da implementação de cursos sobre o ensino do uso racional de medicamentos e de boa prescrição médica.

No Brasil, a grande dificuldade de se implantar um bom serviço de dispensação é a ausência do profissional farmacêutico nos estabelecimentos de saúde. É necessário, portanto, no setor público, maior investimento, por parte dos governantes, na contratação do profissional farmacêutico para melhor condução dos programas de assistência farmacêutica e implementação da atenção farmacêutica nas unidades de saúde, assim como, no setor privado, se exija o cumprimento da Lei $n^{\circ} .5 .99124$.

\section{Resumo}

O estudo visa avaliar aspectos da relação médicopaciente e dispensador-paciente, durante a conduta prescritiva e no ato da dispensação de medicamentos, a partir do ponto de vista dos pacientes. Trata-se de um estudo transversal de base populacional realizado em Fortaleza, Ceará, Brasil, no qual foram utilizadas informações de 957 pessoas, sendo que 904 responderam sobre a última consulta médica; e 831, sobre a última dispensação. As informações coletadas dizem respeito às práticas desses profissionais e do comportamento do paciente, no que diz respeito às perguntas e informações formuladas para melhor direcionar a escolha e o consumo dos medicamentos. Na escolha da terapêutica, o médico falha ao não perguntar sobre alergias medicamentosas anteriores (65\%) e uso de outros medicamentos (64,1\%), e menospreza, na maioria das vezes, os cuidados com as possiveis reações adversas $(73,3 \%)$ e interações medicamentosas $(58,2 \%)$. $\mathrm{Na}$ dispensação do medicamento, a situação é ainda mais grave, pois ela é realizada, principalmente, pelos balconistas $(57,1 \%)$. O estudo sugere a baixa qualidade no atendimento médico e farmacêutico e a apatia do paciente no processo que envolve a prescrição e dispensação de medicamentos e seu uso racional.

Prescrição de Medicamentos; Dispensário de Medicamentos; Uso de Medicamentos; Farmácias

\section{Colaboradores}

P. S. D. Arrais participou na concepção, desenho, coleta, organização do banco de dados, análise e interpretação dos dados e redação do artigo. M. L. Barreto colaborou na análise, interpretação e discussão dos resultados. H. L. L. Coelho participou na concepção e elaboração do projeto, desenvolvimento dos instrumentos, interpretação e discussão dos resultados. Todos os autores colaboraram na confecção do artigo.

\section{Agradecimentos}

Este trabalho é parte da Tese de Doutorado em Saúde Pública de P. S. D. Arrais, apresentada ao Instituto de Saúde Coletiva da Universidade Federal da Bahia, em fevereiro de 2004, com apoio da Fundação Cearense de Amparo à Pesquisa (processo no. 231/02), da Farmácia Escola da Universidade Federal do Ceará e do Conselho Nacional de Desenvolvimento Científico e Tecnológico. Agradecimentos especiais ao Dr. Francisco José Moreira Lopes, chefe da Unidade Estadual do Instituto Brasileiro de Geografia e Estatística no Ceará, às famílias entrevistadas e à professora Dr. Mírian Parente Monteiro do Departamento de Farmácia da Universidade Federal do Ceará, pela revisão do texto. 


\section{Referências}

1. Organização Mundial da Saúde. Guia para a boa prescrição médica. Porto Alegre: Editora Artmed; 1998.

2. Conselho Federal de Farmácia. Código de ética da profissão farmacêutica. Diário Oficial da União 2004; 17 nov.

3. Prefeitura Municipal de Fortaleza. Plano municipal de assistência farmacêutica. Fortaleza: Célula de Assistência Farmacêutica, Coordenadoria de Políticas e Saúde, Secretaria Municipal de Saúde; 2003.

4. Correia LL. Atenção em saúde e eqüidade: uma análise de duas microrregiões assistenciais de saúde do Estado do Ceará. Rev Med Univ Fed Ceará 2000; 40:57-76.

5. Cunha-da-Silva CD, Coelho HLL. Centro e informações sobre medicamentos: auxiliando a decisão terapêutica. Giornale Italiano di Farmacia Clinica 1999; 13:93-102.

6. Piccini RX, Victora CG. How well is hypertension managed in the community? A population-based survey in a Brazilian city. Cad Saúde Pública 1997; 13:595-600.

7. Instituto Brasileiro de Defesa do Consumidor. Faltam remédios essenciais no SUS. Consumidor SA 2003; 69:14-9.

8. Gerência Geral de Regulação Econômica e Monitoramento do Mercado, Agência Nacional de Vigilância Sanitária. Disponibilidade de medicamentos genéricos: Fortaleza e Interior do Ceará. Brasília: Gerência Geral de Regulação Econômica e Monitoramento do Mercado, Agência Nacional de Vigilância Sanitária; 2003.

9. Arrais PSD, Brito LL, Barreto ML, Coelho HLL. Prevalência e fatores determinantes do consumo de medicamentos no Município de Fortaleza, Ceará, Brasil. Cad Saúde Pública 2005; 21:1737-46.

10. Secretaria Municipal de Desenvolvimento Social. Indicadores básicos. Boletim de Saúde de Fortaleza 2000; 4:7-15.

11. Conselho Nacional de Secretários de Saúde. A saúde na opinião dos brasileiros. Brasília: Conselho Nacional de Secretários de Saúde; 2003.

12. Fernandes MEP. A utilização de medicamentos na atenção à saúde, em nível domiciliar, no Município de Fortaleza [Dissertação de Mestrado]. Fortaleza: Departamento de Saúde Comunitária, Universidade Federal do Ceará; 1998.

13. Mosegui GBG, Rozenfeld S, Veras RP, Vianna CMM. Avaliação da qualidade do uso de medicamentos em idosos. Rev Saúde Pública 1999; 33:437-44.
14. Conselho Federal de Medicina. Código de Ética Médica. Diário Oficial da União 1988; 8 jan.

15. Franco ALS. A relação médico-paciente no contexto do Programa de Saúde da Família. Um estudo observacional em três municípios baianos [Tese de Doutorado]. Salvador: Instituto de Saúde Coletiva, Universidade Federal da Bahia; 2002.

16. Baos VV. La calidad en la prescripción de medicamentos. Inf Ter Sist Nac Salud 1999; 23:45-54.

17. Silva AS. Cotidiano médico. Verso e reverso da relação médico-paciente. São Luís: Edufma, 2002.

18. Machado MH, Pereira P. Los recursos humanos y el sistema de salud en Brasil. Gac Sanit 2002; 16:8993.

19. Paes-da-Silva MJ. O papel da comunicação na humanização da atenção à saúde. Bioética 2002; 10:73-85.

20. Bakirtzief Z. Identificando barreiras para aderência ao tratamento de hanseníase. Cad Saúde Pública 1996; 12:497-505.

21. Prefeitura Municipal de Fortaleza. Investigação do uso de medicamentos nos serviços de saúde do Município de Fortaleza-CE. Fortaleza: Núcleo de Assistência Farmacêutica, Coordenadoria de Saúde, Secretaria Municipal de Desenvolvimento Social; 2000.

22. Pepe VLE, Castro CGSO. A interação entre prescritores, dispensadores e pacientes: informação compartilhada como possível benefício terapêutico. Cad Saúde Pública 2000; 16:815-22.

23. Departamento de Atenção Básica, Secretaria de Políticas de Saúde, Ministério da Saúde. Política nacional de medicamentos. Brasília: Secretaria de Políticas de Saúde, Ministério da Saúde; 2001.

24. Brasil. Lei no. 5.991, de 17 de dezembro de 1973. Dispõe sobre o controle sanitário do comércio de drogas, medicamentos, insumos farmacêuticos e correlatos, e dá outras providências. Diário Oficial da União 1973; 21 dez.

25. Brasil. Portaria no. 344. Aprova o Regulamento Técnico sobre substâncias e medicamentos sujeitos a controle especial. Diário Oficial da União 1998; 12 mai.

26. Departamento de Gestão e de Educação na Saúde, Secretaria de Gestão do Trabalho e da Educação na Saúde, Ministério da Saúde. Política de educação e desenvolvimento para o SUS. Caminhos para a educação permanente em saúde. Pólos de educação permanente em saúde. Brasília: Ministério da Saúde; 2003.

Recebido em 09/Ago/2005

Versão final reapresentada em 27/Jul/2006

Aprovado em 18/Ago/2006 